\title{
Doping high Tc superconductors with oxygen and metallic atoms: a molecular dynamics study
}

\author{
Stoll, Erich ; Stern, Christian ; Singer, Johannes ; Stucki, Peter
}

\begin{abstract}
Using classical molecular dynamics based on Lennard-Jones-like potentials, a mechanically stable YBa2Cu3O7 high Tc superconductor structure is generated. This process is controlled via interactive computer graphics. After doping atoms into or removing atoms from the sample using a recently implemented picking mechanism, the lattice oscillation energy is annihilated with a simulated annealing procedure. The remaining minimum ground state energy allows marking of the preferred doping location. Information on the doping mechanism is important because the magnetic and superconducting properties of these compounds depend very strongly on their oxygen content
\end{abstract}

DOI: https://doi.org/10.1557/jmr.1997.0384

Posted at the Zurich Open Repository and Archive, University of Zurich ZORA URL: https://doi.org/10.5167/uzh-154564

Journal Article

Published Version

Originally published at:

Stoll, Erich; Stern, Christian; Singer, Johannes; Stucki, Peter (1997). Doping high Tc superconductors with oxygen and metallic atoms: a molecular dynamics study. Journal of Materials Research, 12(11):29012906.

DOI: https://doi.org/10.1557/jmr.1997.0384 


\title{
Doping high $T_{c}$ superconductors with oxygen and metallic atoms: A molecular dynamics study
}

\author{
Erich Stoll \\ Physik-Institut, Universität Zürich-Irchel, Winterthurerstr. 190, CH-8057 Zürich, Switzerland \\ Christian Stern \\ Institut für Informatik der Universität Zürich, MultiMedia Laboratorium, Winterthurerstr. 190, \\ CH-8057 Zürich, Switzerland \\ Johannes Singer \\ Physik-Institut, Universität Zürich-Irchel, Winterthurerstr. 190, CH-8057 Zürich, Switzerland \\ Peter Stucki \\ Institut für Informatik der Universität Zürich, MultiMedia Laboratorium, Winterthurerstr. 190, \\ CH-8057 Zürich, Switzerland
}

(Received 11 April 1997; accepted 6 August 1997)

Using classical molecular dynamics based on Lennard-Jones-like potentials, a mechanically stable $\mathrm{YBa}_{2} \mathrm{Cu}_{3} \mathrm{O}_{7}$ high $T_{c}$ superconductor structure is generated. This process is controlled via interactive computer graphics. After doping atoms into or removing atoms from the sample using a recently implemented picking mechanism, the lattice oscillation energy is annihilated with a simulated annealing procedure. The remaining minimum ground state energy allows marking of the preferred doping location. Information on the doping mechanism is important because the magnetic and superconducting properties of these compounds depend very strongly on their oxygen content.

\section{INTRODUCTION}

In materials research, small composition changes away from known compounds are desired. Frequently, this "small" deviation is accompanied by rather dramatic structural changes, especially if the system is close to phase changes. They may strongly influence several other physical properties.

Examples include doped semiconductors, which are of great technological importance; another prominent example in solid state physics is high-temperature superconducting copper oxides. Here, the starting point is a layered perovskite $\mathrm{CuO}_{2}$ crystal, usually intercalated with rare earth ions. Examples are $\mathrm{La}_{2} \mathrm{CuO}_{4}$ or $\mathrm{YBa}_{2} \mathrm{Cu}_{3} \mathrm{O}_{6}$. These substances are doped by a variation of, e.g., the rare earth ion concentration or the oxygen content. Surprisingly, these rather small changes of ion fractions $\left\{\right.$ like the oxygen variation from $\mathrm{YBa}_{2} \mathrm{Cu}_{3} \mathrm{O}_{6}$ to $\mathrm{YBa}_{2} \mathrm{Cu}_{3} \mathrm{O}_{7}$ [upper left corner in Fig. 1 and Fig. 2(a)]\} cause dramatic physical and electronic changes.

The system undergoes a transition from an antiferromagnetically ordered insulator via a superconductor to a normal metal. In conjunction with the change in the transport properties, less well-known structural changes also take place. Only a few details are known about the exact crystallographic location of the newly introduced atoms. As in the shown compound, the magnetic and superconducting properties of all high $T_{c}$ cuprate fami- lies depend strongly on the dopant concentration of these compounds. ${ }^{1}$

For example, the oxygen content can be controlled by the pressure of the ambient oxygen atmosphere in conjunction with the temperature of synthesis and sample annealing. Unfortunately, only very few details are known about the microscopic dynamics of such processes. We propose an approach to elucidate this problem using computer simulations. In principle, to generate reliable interatomic interactions to investigate the system dynamics and stability, $a b$ initio quantum chemistry calculations should be used. Unfortunately, also with today's supercomputers the size of such systems is restricted to only a few unit cells, and the complicated electronic structure does not allow an all-electron calculation. Therefore, pseudopotentials have to be applied. This is performed, e.g., in the software packages developed by Car and Parrinello ${ }^{2}$ and Stumpf and Scheffler. ${ }^{3}$ Their computer codes allow movement of the atoms into an equilibrium position simultaneously to achieve an electron energy minimization.

The $\mathrm{Cu}-\mathrm{O}$ interaction, based on the interaction and hybridization of $\mathrm{Cu}-3 d$ and $\mathrm{O}-2 p$ electrons, can be calculated correctly only ${ }^{4}$ by an all-electron computation without using pseudopotentials and by considering the electron correlation with the local density approach ${ }^{5}$ or the generalized gradient approximation. ${ }^{6}$ A similar 


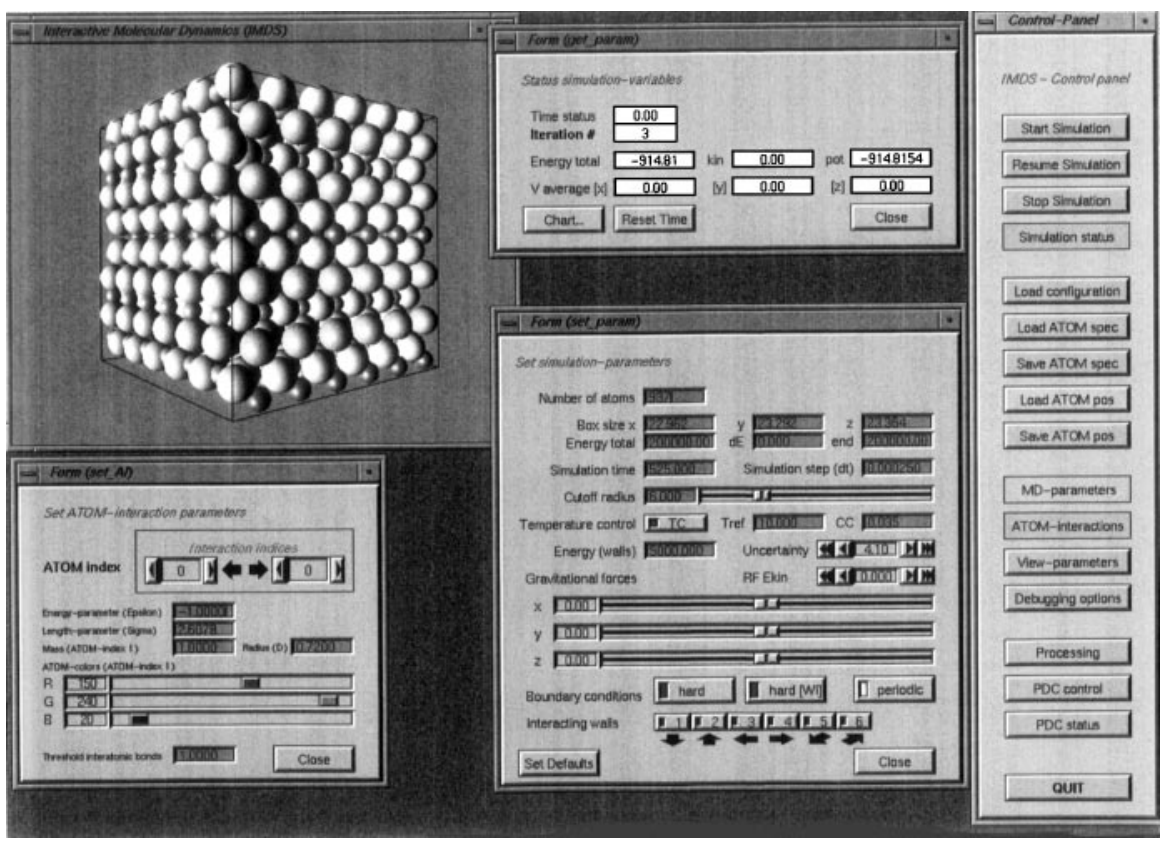

FIG. 1. Screen shot of an interactive simulation session. The panel on the right allows one to start and stop the simulation process and to select a variety of other panels. Using these panels, interaction parameters may be configured and atom positions can be loaded and stored. The simulation process as well as the visualization of the physical system (plotted at the left upper corner of the figure) may be controlled interactively. The panel at the top shows the energetic state of the system, the panel on the left the interaction between the particles, and the panel at the bottom contains elements for adjusting the temperature, the boundary conditions, the box size, and the cutoff radius.

behavior concerning the $\mathrm{Cu}-\mathrm{Cu}$ interaction was already observed in the interpretation of one of the first inelastic neutron scattering results on $\mathrm{Cu}^{7}$ With the pseudopotential formalism the $\mathrm{Cu}-3 d$ electrons do not contribute to the interatomic interaction. To calculate the phonon frequencies of the dispersion curves correctly, a phenomenological Born-Meier potential also had to be used. $^{7}$ Therefore, to simulate the observed experimental behavior of the YBCO superconductor family, we propose a different-simplified-potential approach.

Using the Interactive Molecular Dynamics System (IMDS) package, ${ }^{8-10}$ an array of YBCO unit cells is investigated based on a simplified net interaction with repulsive and attractive forces of the type $V\left(d_{i j}\right)=$ $4\left[|\epsilon|\left(\sigma / d_{i j}\right)^{12}-\epsilon\left(\sigma / d_{i j}\right)^{6}\right] . \sigma$ and $\epsilon$ are tunable parameters and $d_{i j}$ denotes the interatomic distance. A proper choice of these parameters allows the identification and reproduction of known stable YBCO structures.

Based on this approach, we are able to study the influence of new metal and oxygen dopant atoms and defects., i.e., the system stability, and by annealing to zero temperature, we can reconstruct the atomic structure and calculate the appropriate ground state energy. Its minimum shows the preferred doping location. Therefore, this method allows an in-depth investigation of the processes involved in the synthesis of new structural compounds and their chemical and structural stability, and it reveals information that contributes to the understanding of structural phase changes.
The simulation using the interactive molecular dynamics system package ${ }^{8,9}$ not only gives a sophisticated on-line visualization of the processes involved in the formation of a certain structure, but it also provides tools for interactive manipulation and control of these largely not-understood chemical and physical processes.

\section{THEORY}

The unknown "true" interaction is replaced by a simplified net interaction. The potential energy can be assumed to be a superposition of two particle potentials $V\left(\mathbf{r}_{\mathbf{i}}, \mathbf{r}_{\mathbf{j}}\right)$. As a first assumption, such potentials should depend only on the absolute value of the two-particle distance $d_{i j}$. For ionic systems three terms are important ${ }^{11}$ :

$$
\begin{aligned}
V\left(d_{i j}\right)= & \frac{q_{i} q_{j} \exp \left(-\alpha d_{i j}\right)}{4 \pi \epsilon_{0} d_{i j}} \\
& +\epsilon_{i j}^{1}\left(\frac{\sigma_{i j}}{d_{i j}}\right)^{n}-\epsilon_{i j}^{2}\left(\frac{\sigma_{i j}}{d_{i j}}\right)^{6} .
\end{aligned}
$$

The first term is due to the Coulomb interaction with the influence constant $\epsilon_{0}$. To suppress very vast computing efforts due to its long-range influence this term is reduced by an exponential function with $\alpha$ as a damping factor. The second and the third terms occur due to the van der Waals core polarization. $\epsilon_{i j}^{1}, \epsilon_{i j}^{2}, \sigma_{i j}$, and the exponent $n$ denote four adjustable parameters.

In a further simplification step, by setting $\alpha=\infty$ the first term is suppressed. The exponent $n$ in the first 
van der Waals term is set to 12 leading to the simple Lennard-Jones potential that was applied with success to simulate heavier rare gas systems. ${ }^{12}$ By choosing a finite number of different atom types, the indices $i$ and $j$ in $\epsilon_{i j}$ and $\sigma_{i j}$ are replaced by $l$ and $m$, enumerating these different types. $\epsilon_{l m}^{1}$ should always be positive to guarantee repulsive interactions when two atoms are very close. On the other hand, $\epsilon_{l m}^{2}$ can be negative. Based on these simplifications, a Lennard-Jones-like potential was used ${ }^{12}$ :

$$
V\left(d_{i j}\right)=4\left[\left|\epsilon_{l m}\right|\left(\frac{\sigma_{l m}}{d_{i j}}\right)^{12}-\epsilon_{l m}\left(\frac{\sigma_{l m}}{d_{i j}}\right)^{6}\right] .
$$

The restriction of the repulsive force in Eq. (2) to its value at $d_{i j}=\sigma_{l m}$ when the distance $d_{i j}$ between two particles becomes smaller than $\sigma_{l m}$ prevents the algorithm from numerical instabilities.

With these approximations, the system is rather tolerant against mismatches of the parameter values. As their initial value the smallest distances $d_{l m}$ are used for the parameters $\sigma_{l m}$ between the different atom types $l$ and $m$. For the prefactors $\epsilon_{l m}$, the sign was chosen according to the electrical charge of the ions. Their absolute values are taken as one.

To determine the parameters, the system has to be stabilized and the particles must be fixed at the locations given by the known atomic structure. In a system with four unit cells (two cells concatenated in the $x$ - and the $y$-direction) the particle velocities are zero before the molecular-dynamics process is switched on. After a few time steps this process is stopped. The particle positions and velocities are saved. By visual inspection the largest velocities are searched for. Then, the parameters acting on these particles are modified. Iteratively, this procedure is repeated until all velocities remain small.

The interaction parameters are listed in Tables I and II. It should be noted, however, that by considering only the stability of the crystal structure, the relative values of $\epsilon_{l m}$ and not their absolute values can be determined. For such an evaluation, additional information is needed, e.g., the elastic constants or phonon dispersion curves. With these parameters, larger systems, e.g., $6 \times 6 \times 2$ unit cells, are simulated. Such a large cell composed of 72 yttrium, 144 barium, 504 oxygen, and 216 copper atoms should guarantee only weak perturbations by doping one atom into or removing one atom from the system. Simulated annealing is applied to force these systems to their ground states. Heating these systems to higher temperatures by multiplying the particle velocities by a certain factor and keeping the temperatures constant by a thermostat ${ }^{8}$ reveals that the structures are rather resistant to internal and external perturbations.
TABLE I. Interaction parameter $\epsilon_{l m}$ in arbitrary units of Eq. (2) between the atom groups $l$ and $m$. These groups are named according to the different elements and positions. The locations of these atoms in the unit cell are plotted in Fig. 2(a).

\begin{tabular}{lrrrrrrr}
\hline \hline & $\mathrm{Cu}(1)$ & $\mathrm{Cu}(2)$ & $\mathrm{O}(1)$ & $\mathrm{O}(2)$ & $\mathrm{O}(4)$ & $\mathrm{Ba}$ & $\mathrm{Y}$ \\
\hline $\mathrm{Cu}(1)$ & -1.0 & -1.00 & 3.0 & 3.0 & 3.0 & 2.0 & 0.30 \\
$\mathrm{Cu}(2)$ & -1.0 & -0.50 & 3.0 & 3.0 & 4.0 & 2.0 & -0.13 \\
$\mathrm{O}(1)$ & 3.0 & 3.00 & -1.5 & -1.0 & -1.0 & 4.0 & 4.00 \\
$\mathrm{O}(2)$ & 3.0 & 3.00 & -1.0 & -0.5 & -1.0 & 4.0 & 4.00 \\
$\mathrm{O}(4)$ & 3.0 & 4.00 & -1.0 & -1.0 & -1.5 & 6.0 & 6.00 \\
$\mathrm{Ba}$ & 2.0 & 2.00 & 4.0 & 4.0 & 6.0 & -1.0 & -1.00 \\
$\mathrm{Y}$ & 0.3 & -0.13 & 4.0 & 4.0 & 6.0 & -1.0 & -1.50 \\
\hline \hline
\end{tabular}

TABLE II. Interaction parameter $\sigma_{l m}$ in angstrom units.

\begin{tabular}{lccccccc}
\hline \hline & $\mathrm{Cu}(1)$ & $\mathrm{Cu}(2)$ & $\mathrm{O}(1)$ & $\mathrm{O}(2)$ & $\mathrm{O}(4)$ & $\mathrm{Ba}$ & $\mathrm{Y}$ \\
\hline $\mathrm{Cu}(1)$ & 2.61 & 3.01 & 1.73 & 1.73 & 1.63 & 3.10 & 3.10 \\
$\mathrm{Cu}(2)$ & 3.01 & 2.51 & 2.07 & 1.72 & 2.07 & 3.01 & 2.86 \\
$\mathrm{O}(1)$ & 1.73 & 2.07 & 2.96 & 3.94 & 2.38 & 2.57 & 2.57 \\
$\mathrm{O}(2)$ & 1.73 & 1.72 & 3.94 & 2.89 & 2.87 & 2.63 & 2.13 \\
$\mathrm{O}(4)$ & 1.63 & 2.07 & 2.38 & 2.87 & 3.28 & 2.45 & 2.45 \\
$\mathrm{Ba}$ & 3.10 & 3.01 & 2.57 & 2.63 & 2.45 & 3.86 & 3.27 \\
$\mathrm{Y}$ & 3.10 & 2.86 & 2.57 & 2.13 & 2.45 & 3.27 & 3.86 \\
\hline \hline
\end{tabular}

\section{DOPING WITH OXYGEN AND METAL ATOMS}

To disentangle the rather complicated structure of the high $T_{c}$ superconductor $\mathrm{YBa}_{2} \mathrm{Cu}_{3} \mathrm{O}_{7}$, the unit cell, plotted in Fig. 2(a), can be subdivided by mirror planes. The first mirror planes are on the top and bottom of the unit cell and contain the $\mathrm{O}(1)$ and the $\mathrm{Cu}(1)$ atom chains. As the unit cell can be concatenated by itself to form the system, both mirror planes are identical. The second mirror plane cutting the unit cell into two equal parts contains the $\mathrm{Y}$ atom and is marked by a dashed rhombus. The largest amount of empty space where additional atoms may have a chance to be absorbed are on these mirror planes. One oxygen or metal atom $(\mathrm{Ba}, \mathrm{Cu}, \mathrm{Y})$ can be deposited on the first plane at $X_{b r}$. Due to the periodic continuity of the unit cell with itself, $X_{b r}$ is also between the lower $\mathrm{Ba}$ atom of this cell and the upper $\mathrm{Ba}$ atom of the cell below it. In Fig. 2(b) this situation is sketched as "bridge." A second position is marked on the top mirror plane in Fig. 2(a) by $X_{c r}$. This point lies between two $\mathrm{Cu}(1)$ atoms and also, via the periodic concatenation of the unit cells, between four Ba atoms, noted as "cross" in Fig. 2(b). The piercing point $X_{c h}$ of the connection line between two $\mathrm{Cu}(2)$ atoms through the first mirror plane is the third location for adsorbing one atom. In Fig. 2(b) it is marked by "chain."

To locate an oxygen or a metal atom at the positions $X_{b r}, X_{c r}$, or $X_{c h}$, a recently developed picking method is applied. ${ }^{13}$ The upper left corner in Fig. 1 shows the threedimensional visualization of a specific particle system (O cross) using the IMDS application. Applying the 

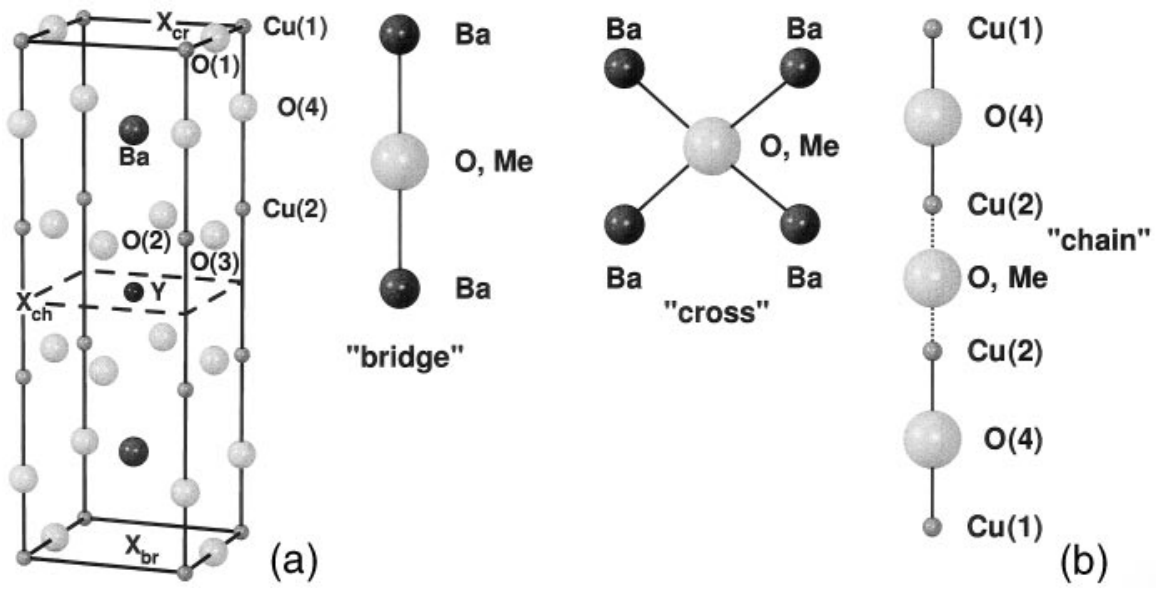

(b)

FIG. 2. (a) Plot of the unit cell of the $\mathrm{YBa}_{2} \mathrm{Cu}_{3} \mathrm{O}_{7}$ high $T_{c}$ superconductor. For the calculations the oxygen atoms $\mathrm{O}(2)$ and $\mathrm{O}(3)$ are assumed identical. (b) "O, Me" between $\mathrm{Ba}$ and $\mathrm{Cu}$ atoms means that either an oxygen atom or a metal atom (Ba, $\mathrm{Cu}$, or $\mathrm{Y})$ has been supplied as a dopant.

mouse pointer, a specified pixel may be selected. When pushing the left mouse key, the particle that contains the specified pixel is selected, and a panel displaying the identification number and coordinates of this particle is activated.

To suppress the ambiguity that may occur when multiple particles are superimposed on the same area, the particles should be plotted as single pixels. After the picking process, the selected particle can be positioned within the given space using the panel mentioned above. Afterward, the new coordinates of this particle are saved and ready for further processing.

As the new positions of the particle are not optimal, their potential energies are rather high (see Table III). Applying the IMDS package,${ }^{8-10}$ lattice oscillations are created. By activating the simulated annealing procedure these oscillations vanish and new stable structures are established.

Another method for determining the most stable equilibrium position is to bombard the system with one oxygen atom carrying a rather high kinetic energy (some orders of magnitude larger than the interaction parameters $\epsilon_{l m}$ denoted in Table I). After switching on the MD algorithm and the simulated annealing procedure, this atom is stopped after a short distance of a few atom diameters at the cross position $X_{c r}$.

To discuss the relative stability and the most preferred position, we have calculated the total ground state energy of our 936 particle system before doping, immediately after we have introduced one additional atom $(\mathrm{Y}, \mathrm{Ba}, \mathrm{Cu}$, and $\mathrm{O})$ at the positions $X_{b r} X_{c r}$, or $X_{c h}$, after the environmental atoms of the doping atom have been relaxed, and immediately after we have taken away this doping atom from the relaxed environment.

As shown in Table III, after relaxation of the environment atoms, the lowest ground state energy for oxygen is that of the cross position $X_{c r}$. This agrees with the bombardment simulation where the oxygen atom has also itself taken this position. Qualitatively, this can be understood by the argument that there is more space left and that at this position the nearest neighbors are the attractive $\mathrm{Cu}(1)$ atoms against the repulsive $\mathrm{O}(1)$ atoms on the bridge position. The chain position is more favorable than the bridge position. The third column in Table III shows that in the relaxed configurations the energy decreases nearly always by reextracting the added atoms. However, only in the cross position the energy increases by extracting the added oxygen atom. This is also an indication that this position is more stable and oxygen atoms may there be bound.

TABLE III. Ground state energies of the $\mathrm{YBa}_{2} \mathrm{Cu}_{3} \mathrm{O}_{7}$ high $T_{c}$ superconductor prior to doping and after depositing one additional atom. "After deposition" means the total (potential) energy immediately after the dopant atom has been inserted. "Relaxed": part of this energy is transformed to lattice vibrational energy and has been removed by the simulated annealing procedure. "Relaxed and dopant reextracted": after the simulated annealing procedure had been performed the additional atom has been removed again. Cross (see Fig. 2) means the position $X_{c r}$ in the middle of four Ba atoms (for oxygen one final simulation state is shown in the system plot in the upper left corner of Fig. 1), bridge means the position $X_{b r}$ between two Ba atoms, and chain means the position $X_{c h}$.

\begin{tabular}{lccc}
\hline \hline \multicolumn{1}{c}{ Position } & $\begin{array}{c}\text { After } \\
\text { deposition }\end{array}$ & Relaxed & $\begin{array}{c}\text { Relaxed and } \\
\text { dopant reextracted }\end{array}$ \\
\hline Prior doping & -950.10 & -950.10 & -950.10 \\
O cross & -947.24 & -950.27 & -948.35 \\
O bridge & -584.78 & -901.11 & -916.86 \\
O chain & -590.06 & -906.35 & -931.70 \\
Y cross & -864.33 & -896.40 & -913.24 \\
Y bridge & -472.75 & -831.89 & -858.12 \\
Y chain & -685.68 & -817.19 & -866.02 \\
Ba cross & -526.28 & -767.22 & -832.67 \\
Ba bridge & -391.54 & -688.48 & -763.62 \\
Cu cross & -790.66 & -916.04 & -926.47 \\
\hline \hline
\end{tabular}


For additional metal atoms $(\mathrm{Y}, \mathrm{Ba}$, and $\mathrm{Cu})$ the situation seems to be more difficult, but also there the cross position is preferred, creating at least metastable configurations. The chain position could be achieved only by the $\mathrm{Y}$ atom. The bridge position is very unstable. Small perturbations have moved one $\mathrm{Cu}$ and one $\mathrm{Ba}$ atom during the MD simulation to the cross position.

\section{SUMMARY AND CONCLUSIONS}

A stable structure of a $\mathrm{YBa}_{2} \mathrm{Cu}_{3} \mathrm{O}_{7}$ high $T_{c}$ superconductor has been created by using the Interactive Molecular Dynamics System (IMDS) package ${ }^{8,9}$ and Lennard-Jones-like potentials ${ }^{12}$ (see Fig. 3). These simple potentials were chosen because ab initio quantum chemistry all-electron calculations cannot yet be performed for large enough clusters even with today's supercomputers.

Inserted new oxygen and metal atoms increase the energy. This additional energy is dissipated by lattice oscillations and reduced by a simulated annealing procedure $^{8}$ that cools the system to very low temperatures. The remaining ground state energies are shown in Table III. Their minimum denotes the most stable dopant position.
Because the magnetic and superconducting properties of these compounds depend very strongly on their oxygen content, ${ }^{1}$ we may now understand what happens when the pressure of the ambient oxygen atmosphere is increased and the bombardment of the sample with oxygen atoms is enforced.

This simulation not only offers a sophisticated online visualization of the processes involved in the formation of a certain structure, but also provides tools for interactive manipulation and control of these largely nonunderstood chemical and physical processes. In addition to the known experimental techniques using local probes [Nuclear Magnetic Resonance ${ }^{14}$ (NMR), Nuclear Quadrupole Resonance ${ }^{14}$ (NQR), Scanning Tunneling Microscopy $^{15}$ (STM), and Atomic Force Microscopy ${ }^{16}$ (AFM)], our results stimulate the demand for new experimental methods to investigate more favorably the neighborhood of the doped atoms.

Due to the fact that today's supercomputers are not powerful enough to perform all-electron $a b$ initio quantum chemistry calculations for all atoms, new and expanded numerical methods should be applied like, e.g., the one ${ }^{17}$ that combines quantum chemistry all-electron calculations $^{18}$ of atoms in a small cage-like region in the neighborhood of the investigated atoms and molecule

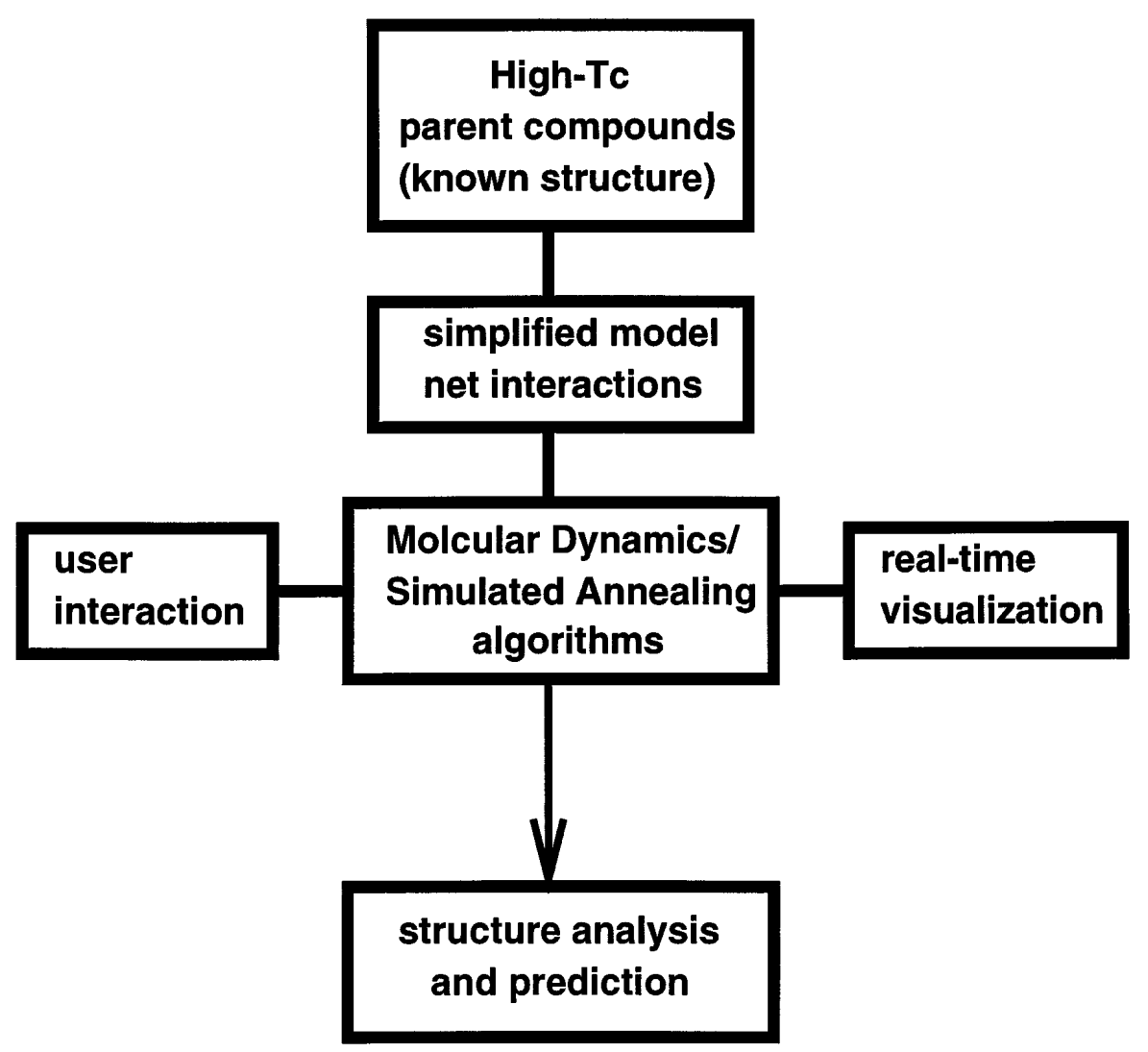

FIG. 3. Schematic work schedule of our approach: Interaction and visualization allow on-line control and manipulation of this structure optimization. Although less is known about the microscopic details, this procedure provides rather detailed and good results. 
mechanical computations with simple potentials ${ }^{19}$ on all other atoms.

\section{ACKNOWLEDGMENT}

We are grateful to the Swiss National Science Foundation, Priority Program Computer Science, for financial support.

\section{REFERENCES}

1. For a review, see, e.g., T. Schneider and H. Keller, Int. J. Mod. Phys. B 5, 487 (1993) and references therein.

2. R. Car and M. Parrinello, Phys. Rev. Lett. 55, 2471 (1985).

3. R. Stumpf and M. Scheffler, Computer Phys. Commun. 79, 447 (1994).

4. P. Hüsser, E. Stoll, H. U. Suter, and P. F. Meier, unpublished.

5. S. H. Vosko, L. Wilk, and M. Nussair, Can. J. Phys. 58, 1200 (1980).

6. A. D. Becke, Phys. Rev. A 38, 3098 (1988).

7. T. Schneider and E. Stoll, Solid State Commun. 4, 79 (1966).
8. Christian Stern, Peter Stucki, and Erich Stoll, in Proceedings Information Conference Module 3 Massively Parallel Systems, edited by K. Bauknecht and J. M. Grossenbacher (Swiss Nat. Sci. Found., Bern, 1994), p. 29.

9. Christian Stern, Peter Stucki, and Erich Stoll, unpublished.

10. Christian Stern, Internet: http://www.ifi.unizh.ch/staff/stern.html.

11. J. Ch. Schön and M. Jansen, Angew. Chem. 108, 1358 (1996).

12. L. Verlet, Phys. Rev. 159, 98 (1967).

13. Christian Stern, Peter Stucki, and Erich Stoll, unpublished.

14. For a review, see D. Brinkmann and M. Mali, NMR Basic Principles and Progress (Springer, Heidelberg, 1994), Vol. 31, p. 171.

15. G. Binning, H. Rohrer, Ch. Gerber, and E. Weibel, Appl. Phys. Lett. 40, 178 (1982).

16. G. Binning, C.F. Quate, and Ch. Gerber, Phys. Rev. Lett. 56, 930 (1986).

17. Tiziana Mordasini Denti and Christoph Hanser, CrosSCutS 6 (3), 19 (1996)

18. M. J. S. Dewar and W. Thiel, J. Am. Chem. Soc. 99, 4899 (1977).

19. W. F. van Gunsteren and H. J. C. Berendsen, GROningen MOlecular Simulation package and manual (GROMOS) (Biomos, University of Groningen, 1987). 